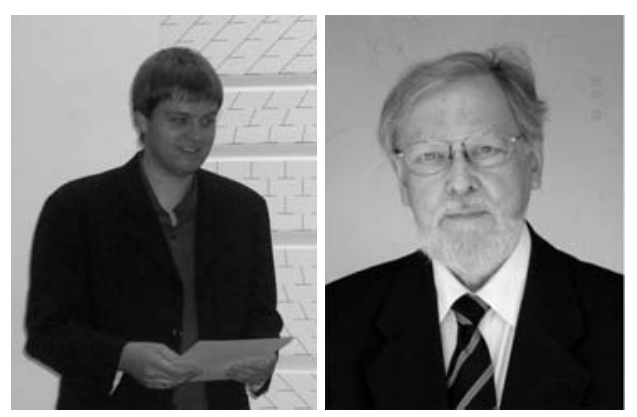

Prof. Otmar Venjakob und Prof. Erwin Stein

(Photos: DFG)

\title{
Von Kaven-Preis für Mathematik
}

Professor Otmar Venjakob und Professor Erwin Stein sind die ersten Preisträger des neu geschaffenen und von der Deutschen Forschungsgemeinschaft (DFG) verliehenen von Kaven-Preises für Mathematik. (DFG)

Bei einer Feierstunde am 14. Oktober 2005 im Arithmeum in Bonn wurde Otmar Venjakob, der erst kürzlich aus dem Heisenberg-Programm der DFG auf eine Professur an die Universität Bonn berufen wurde, für seine besonderen wissenschaftlichen Leistungen mit dem von Kaven-Ehrenpreis sowie 10000 Euro Preisgeld ausgezeichnet. Erwin Stein ist Preisträger des ebenfalls erstmals vergebenen und mit insgesamt 5000 Euro dotierten von Kaven-Förderpreises.

Otmar Venjakob erhält als erster Preisträger von Wolfgang Dahmen, Mitglied des Senats der DFG, den neuen von Kaven-Ehrenpreis für Mathematik.

Beide Preise finanzieren sich aus einer im Dezember 2004 von dem Detmolder Mathematiker Herbert von Kaven und der DFG ins Leben gerufenen Stiftung. Der Stifter und Namensgeber des ersten MathematikPreises, den die DFG vergibt, ist mit 97 Jahren selbst immer noch aktiv forschender Mathematiker mit einem besonderen Faible für die Grundlegungen der Mathematik. Seine Vorliebe für konstruktive und instrumentale Methoden bestimmt auch die Ausrichtung des Förderpreises, welcher der Unterstützung von Vorhaben im Bereich der instrumentalen Mathematik dienen soll.

Zentral wirkt bei der Auswahl der Preisträger das Fachkollegium Mathematik der DFG mit. Die von der Wissenschaft gewählten Vertreterinnen und Vertreter der in Deutschland arbeitenden Forschungsmathematiker treffen die Auswahlentscheidung für den Preisträger des Ehrenpreises und sprechen die Empfehlung für zu fördernde Vorhaben auf dem Gebiet der instrumentalen Mathematik aus. Die aktuellen Mitglieder des Fachkollegiums sowie dessen bisherige Vorsitzende haben dabei zusammen mit den Gremienmitgliedern der DFG für die Mathematik auch das Vorschlagsrecht für mögliche Preisträger des von Kaven-Ehrenpreises, für den eine Selbstbewerbung nicht möglich ist. Dieser Preis soll, sofern keine weiteren geeigneten Vorschläge vorliegen, dem besten Heisenberg-Stipendiaten in der Mathematik aus dem jeweils vergangenen Jahr als besondere Auszeichnung zuerkannt werden - sozusagen als i-Tüpfelchen für dieses bei Mathematikern besonders begehrte und prestigeträchtige Programm.

Der erste Träger des von Kaven-Ehrenpreises erfüllt alle Bedingungen, die an einen würdigen Preisträger gestellt werden können. Otmar Venjakob ist nach nur wenigen Monaten als Heisenberg-Stipendiat zum Beginn des Wintersemesters 2005/06 auf eine Professur an die Universität Bonn berufen worden. Er arbeitet auf dem seit einigen Jahren mit ganz neuen Ergebnissen aufwartenden Gebiet der nicht-kommutativen Iwasawa-Theorie, einem Teilgebiet der algebraischen Zahlentheorie beziehungsweise arithmetischen Geometrie, und gehört zu den dieses Gebiet derzeit prägenden Wissenschaftlern. Otmar Venjakob wurde für seine bahnbrechenden Arbeiten unter anderem bereits im Jahr 2004 in Stockholm mit dem EMS Preis der European Mathematical Society ausgezeichnet.

Förderpreisträger Erwin Stein aus Hannover ist international anerkannter Vorreiter der Baumechanik und der Numerischen Mechanik, Gründungs-direktor des Instituts für Baumechanik und Numerische Mechanik (IBNM) in Hannover und leitete dieses Institut bis zu seiner Emeritierung im Jahr 1998 über fast 30 Jahre. Er beschäftigt sich neben seiner vielfältigen Forschungstätigkeit im Bereich Computational Mechanics ebenfalls mit der Konstruktion und dem Nachbau historischer Rechenmaschinen nach Gottfried Wilhelm Leibniz. Zur Unterstützung dieser Arbeiten hat ihm das Fachkollegium Mathematik den von Kaven-Förderpreis 2005 zuerkannt.

Weitere Informationen unter http://www.dfg. de/aktuelles_presse/preise/von_kaven_preis/ index.html. 